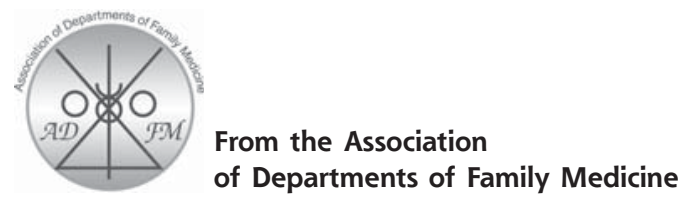

Ann Fam Med 2005;3:93. DOI: 10.1370/afm.279.

\section{SERVING NATIVE AMERICAN COMMUNITIES}

Academic departments have a shared purpose to better serve the local community, to bring diversity to our experience in training sites, and to reflect the diversity of those who reside in our states. Nowhere is that mission better demonstrated than in the departments of family medicine based in states with large Native American populations. These departments have developed innovative programs to reach and collaborate with members of their Native American communities.

The University of North Dakota (UND) School of Medicine and Health Sciences Department of Family Medicine has residency programs in Bismarck and Minot involved with their local reservations. Faculty and residents from Minot travel weekly to provide maternity and women's health services to the Fort Berthold area. Both residency groups provide inpatient services at their main teaching hospitals. Several of the residents have gone on to work in these sites, which is one goal of the program. In addition, medical students have family medicine clinical experiences on the reservations at Indian Health Service (IHS) sites. Similarly, the physician assistant (PA) program is working to attract students from the Native populations, enhance the PA student training in cultural issues, and improve care to Native American elders through a cooperative educational program with local elders.

The university regularly admits 7 Native American students yearly through the Indians into Medicine (INMED) Program 5 students stay in North Dakota, and 2 go to the University of South Dakota (USD) for their clinical education. UND is also the recipient of a telemedicine grant to develop an annual eye screening program for Native Americans with diabetes.

The University of New Mexico Department of Family and Community Medicine has a long-standing commitment to serving Indian people. Three of the 5 founding department faculty worked in the IHS, and today, of the 46 faculty in the department, $28 \%$ have worked or are working with the IHS and 4 (9\%) are American Indian. Strong links have been established between the Department and the Albuquerque IHS, including faculty appointments for the IHS physicians. Department faculty provide health services in rural and urban Indian communities, faculty and residents admit referred Indian patients to the department's inpatient service, and residents train in IHS facilities.

Since 1998, 2 of 18 family medicine residents at New Mexico admitted each year are allowed to select the Albuquerque Indian Hospital for their required 3 -year continuity clinic. A review of the outcome of this residency "track" shows that, of the 13 residents who have graduated after 3 years of continuity clinic training at the IHS, 7 (54\%) were American Indian, 9 (69\%) currently work in IHS, tribal, or urban Indian settings $s_{i}$ and $4(31 \%)$ work in non-Indian, underserved rural, urban or migrant health centers. Family medicine residency training in Native Indian communities has benefits for the Indian communities in terms of service, benefits for IHS in terms of recruitment of a stable health workforce, and benefits for the residency in terms of unique educational opportunities.

Finally, the USD School of Medicine and Health Sciences Department of Family Medicine has a central focus of reducing health disparities, especially for the American Indian population. This work is done through medical student rotations in preventive medicine, obstetrics-gynecology, and community health at IHS clinics and hospitals, medical student research projects addressing tribal and IHS priorities, faculty research and service grants/contracts with tribes, and an epidemiology contract through the Center for Rural Health Improvement with the Aberdeen Area IHS. Medical student projects include helping a tribe develop its own cancer plan, conducting a research survey of IHS provider screening habits and training needs for domestic violence, and a follow-up project the next year that provides ACOG domestic violence screening training CME to IHS providers. Department faculty and residents provide community lectures on educational opportunities in medicine and the health sciences to students in the communities where they work.

Like politics, health care is inherently a local issue, and the needs and concerns of local groups are as varied as the groups themselves. Academic departments must adapt to the local environment, build on local strengths, and respond to local needs. The programs described here represent only a small part of the diversity of departmental programs, but they are models of how departments have responded to their local conditions and built programs and systems that reach out to, collaborate with, and help those we seek to serve.

Elizabeth A. Burns, MD, MA

University of North Dakota, Grand Forks Chuck North, MD

University of New Mexico, Albuquerque

Sarab Patrick, MPH, PbD

University of South Dakota, Sioux Falls 